\title{
3D spectral synthesis and rotational line broadening (Research Note)
}

\author{
H.-G. Ludwig \\ GEPI, CIFIST, Observatoire de Paris-Meudon, 5 place Jules Janssen, 92195 Meudon Cedex, France \\ e-mail: Hans .Ludwig@obspm
}

Received 10 December 2006 / Accepted 6 May 2007

\section{ABSTRACT}

\begin{abstract}
Context. Spectral synthesis calculations based on three-dimensional stellar atmosphere models are limited by the affordable angular resolution of the radiation field. This hampers an accurate treatment of rotational line broadening.

Aims. We aim to find a treatment of rotational broadening of a spherical star when the radiation field is only available at a modest number of limb-angles.

Methods. We apply a combination of analytical considerations of the line-broadening process and numerical tests.

Results. We obtain a method which is closely related to classical flux convolution and which performs noticeably better than a previously suggested procedure. It can be applied to rigid as well as differential rotation.
\end{abstract}

Key words. hydrodynamics - radiative transfer - stars: atmospheres - line: profiles - methods: numerical

\section{Introduction}

Spectral synthesis calculations based on time-series of threedimensional (3D) background structures from hydrodynamical model atmospheres are computationally demanding and pose limits on the affordable resolution for representing the angular dependence of the radiation field. In such calculations, the number of employed azimuthal directions typically ranges from four to eight, the number of inclined directions representing the center-to-limb variation being between three and five. Obviously, the resolution is not high, and in particular makes an accurate implementation of rotational line broadening somewhat difficult. To our knowledge, the only published method handling rotational broadening in the $3 \mathrm{D}$ case is that of Dravins $\&$ Nordlund (1990). Here, we describe an alternative procedure which provides higher accuracy at similar computational complexity. It is closely related to standard flux convolution (e.g., Gray 1992). Its development was motivated by the demand for an accurate description of the rotational broadening of spectral lines in the solar spectrum (Caffau et al. 2007).

\section{The problem and basic assumptions}

For the moment, we neglect differential rotation, and treat the star as spherical and rigidly rotating. Local-box hydrodynamical model atmospheres provide a statistical realization of a small patch of the surface flow pattern. Formally, we want to obtain an estimate of the expectation value of a rotationally broadened, disk-integrated line profile. Rotational symmetry with respect to the stellar disk center implies that there is no azimuthal dependence of the radiation field. All we need to know for evaluating the disk-integrated rotationally broadened line profile are temporal and azimuthal averages of the emergent radiation intensity of the local model as a function of asterocentric angle $\vartheta$. Hence, the problem is equivalent to the rotational line broadening problem in standard, plane-parallel model atmospheres.

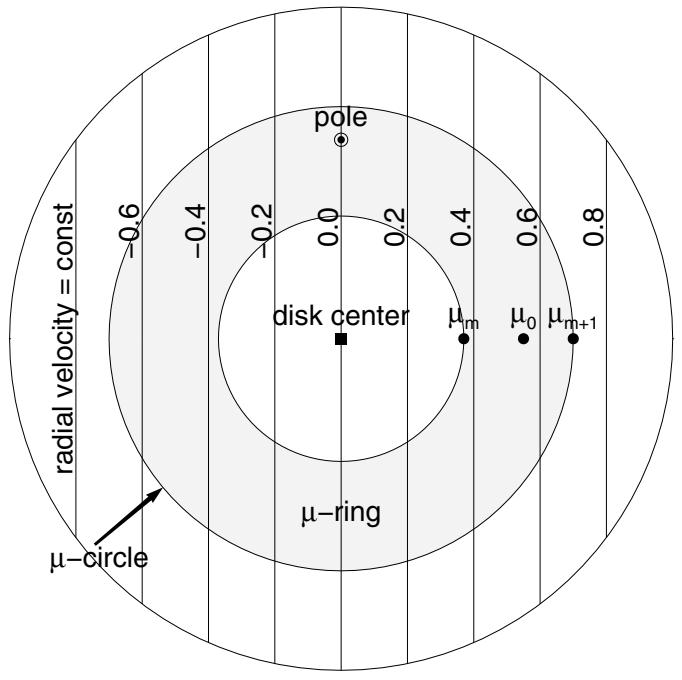

Fig. 1. Illustration of the apparent radial velocity distribution on a stellar disk for solid body rotation: vertical lines of constant radial velocity are labeled by their velocity in units of $V \sin (i)$. They lie parallel to rotational pole - disk center direction. For further explanations see text.

\section{The method of Dravins \& Nordlund}

We start by describing the procedure of Dravins \& Nordlund (1990, Sect. 2.1) who model rotational broadening as a superposition of line profiles at various $\mu \equiv \cos (\vartheta)$, broadened by the velocity field along circles $\mu=$ const around the stellar disk center. We refer to one of these circles as " $\mu$-circle" (see Fig. 1). The broadening effect of the projected rotational velocity along 
a $\mu$-circle located at $\mu=\mu_{m}$ can be expressed as a convolution according to

$\tilde{I}\left(v, \mu_{m}\right)=\frac{1}{\pi} \int_{-\tilde{v}}^{+\tilde{v}} \mathrm{~d} \xi \frac{I\left(v-\xi, \mu_{m}\right)}{\sqrt{\tilde{v}^{2}-\xi^{2}}}$

where we expressed the wavelength-dependence of the intensity $I\left(\lambda, \mu_{m}\right)$ in the line in terms of the Doppler speed $v, I\left(v, \mu_{m}\right) . \tilde{I}$ is the intensity in the line profile broadened under the action of the radial velocity along the considered $\mu$-circle. $\tilde{v}$ is the maximum projected rotational velocity at $\mu_{m}$ given by

$\tilde{v}\left(\mu_{m}\right)=V \sin (i) \sqrt{1-\mu_{m}^{2}}$.

$V \sin (i)$ is the usual projected rotational velocity of the star as a whole. An obvious feature of the convolution expressed by Eq. (1) is the singularity of the integrand at $\xi= \pm \tilde{v}$, i.e., the presence of sharp spikes. One might already suspect at this point that their presence will be apparent in the fully disk-integrated profile. Numerically, one can handle the singularities by analytically integrating the expression over each resolution element assuming a certain functional dependence of $I(v)$ within them, and summing over all contributions in the interval $[-\tilde{v},+\tilde{v}]$. We assumed a constant behavior of $I$ in each resolution element so that the integral of the kernel function over a resolution element stretching over an interval $\left[\xi_{1}, \xi_{2}\right]$ can be conveniently expressed as

$\int_{\xi_{1}}^{\xi_{2}} \frac{\mathrm{d} \xi}{\sqrt{\tilde{v}^{2}-\xi^{2}}}=\arcsin \left(\frac{\xi_{2}}{\tilde{v}}\right)-\arcsin \left(\frac{\xi_{1}}{\tilde{v}}\right)$.

The disk-integrated, rotationally-broadened flux profile $\tilde{F}(v)$ is obtained by integrating $\tilde{I}$ over the disk according to the standard relation

$\tilde{F}(v)=2 \pi \int_{0}^{1} \mathrm{~d} \mu \mu \tilde{I}(v, \mu) \simeq 2 \pi \sum_{m=1}^{N_{\mu}} w_{m} \tilde{I}\left(v, \mu_{m}\right)$.

The second approximate equality is the discrete approximation to the integral employing a total number of $N_{\mu}$ limb-angles of weight $w_{m}$ at positions $\mu_{m}$.

The original implementation of Dravins \& Nordlund did not use a formulation as convolution but a discrete integration over $\mu$-circles using polar coordinates. While mathematically equivalent to Eq. (1), it somewhat obscures the critical role of the most extreme velocities on a $\mu$-circle for the smoothness of the rotationally broadened spectrum.

Figure 2 illustrates the result of a test of Dravins \& Nordlund's procedure. We broadened an artificial Gaussian line profile with a rotational speed $V \sin (i)$ of three times the line's Doppler width. The rotational speed was chosen to be particularly critical. Effects of smaller rotational velocities become less pronounced due to the smoothing by the Gaussian line profile, at larger rotational speeds deviations are less conspicuous since less localized. For the test case we assumed that the relative line shape is independent of $\mu$, and that the intensity in the continuum follows a linear limb-darkening law with a limb-darkening coefficient of 0.6. Hence, standard flux convolution can be applied to obtain the exact disk-integrated line profile. Figure 2 shows the convergence of the numerical approximation towards the exact result with an increasing total number of limb-angles $N_{\mu}$. As evident in the figure, noticeable deviations between the exact and the numerical result are present up to and including $N_{\mu}=3$.

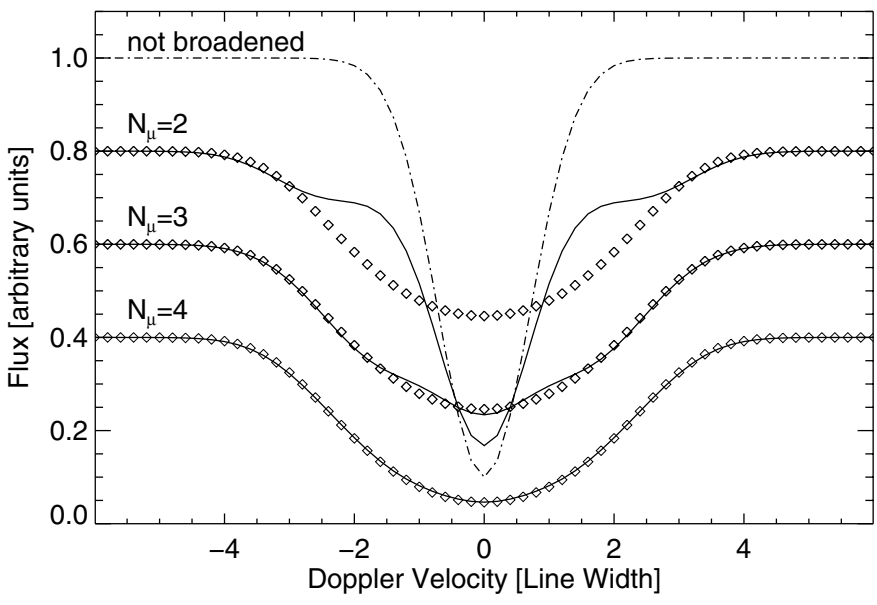

Fig. 2. Rotationally broadened line profiles (solid lines) for different total numbers of asterocentric angles $N_{\mu}$ employing the broadening procedure of Dravins \& Nordlund in comparison to the exact profile (diamonds). For clarity, the broadened line profiles have been vertically offset. Wavelengths are given as Doppler velocities in units of the width of the Gaussian non-broadened profile (dash-dotted line).

\section{An improved method}

The major reason for the "wiggly" behavior of the broadened line profile shown in Fig. 2 are the pronounced spikes in the integrand in Eq. (1). One can reduce their impact by associating a given $I(\mu)$ not only with an infinitely thin $\mu$-circle but with a $\mu$-ring (see Fig. 1 ) of finite extent. The contribution $\tilde{F}(\mu)$ to the rotationally broadened flux profile stemming from the stellar disk area subtended by $\mu \in\left[\mu_{m}, 1\right]$ is given by the convolution

$\tilde{F}\left(v, \mu_{m}\right)=\frac{2}{V^{2} \sin ^{2}(i)} \int_{-\tilde{v}}^{+\tilde{v}} \mathrm{~d} \xi I\left(v-\xi, \mu_{0}\right) \sqrt{\tilde{v}^{2}-\xi^{2}}$.

$\tilde{v}$ is again given by relation (2). Here, we assume that the intensity profile is constant within $\left[\mu_{m}, 1\right]$ represented by the intensity at $\mu=\mu_{0}$ - presumably but not necessarily lying in the interval $\left[\mu_{m}, 1\right]$. Note, that relation (5) takes into account the surface area corresponding to the interval $\left[\mu_{m}, 1\right]$. Its result is a flux-like integral, different from the result of relation (1) which expresses an average intensity since the kernel function is normalized to one. A $\mu$-ring extending over the interval $\left[\mu_{m}, \mu_{m+1}\right]$ can be obtained by subtracting contributions $\left[\mu_{m+1}, 1\right]$ from the contribution of $\left[\mu_{m}, 1\right]$ (assuming $\mu_{m+1}>\mu_{m}$ ) keeping the same intensity at $\mu_{0}$. One can build up the whole visible stellar disk by a number of $\mu$-rings. Their surface area is reflecting the integration weight $w_{m}$ in Eq. (4). As stated before, in each individual ring the intensity is assumed to be $\mu$-independent.

Figure 3 illustrates the outcome of this procedure. A comparison with Fig. 2 shows a more rapid convergence towards the exact result. The improvement is related to the fact that the new method can at least handle exactly the simple case of a globally $\mu$-independent intensity which is not the case for the method of Dravins and Nordlund. One could further refine it by introducing an analytical expression for the $\mu$-dependence of the intensity in each $\mu$-ring - perhaps motivated from a fit to the continuum intensity available at the discrete $\mu_{m}$. However, we did not implement this since from the test it appeared that the accuracy at an affordable number of $N_{\mu}=3$ is already sufficient. There is a caveat to this statement: the accuracy of the methods also hinges on the level of the differential line-shift and -broadening as a function of $\mu$ which we did not test here. This should be checked on a case by case basis. 


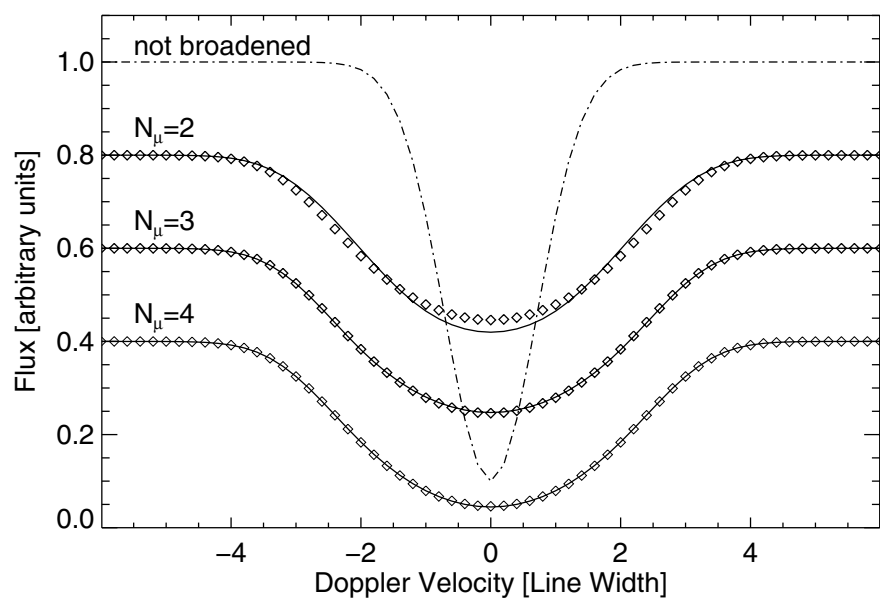

Fig. 3. As for Fig. 2 for our broadening procedure.

\section{Remarks on differential rotation}

Up to this point we considered solid body rotation only. However, the previous discussion made clear that integration over $\mu$-rings should also perform better in the case of differential rotation. The referee made the point that putting efforts into 3D model and spectral synthesis calculations warrants the inclusion of effects of differential rotation to maintain highest level of accuracy. In the following we give a brief demonstration that differential rotation on the level observed in the Sun can indeed be relevant for resulting line profiles. We implemented our method for a case of differential rotation.

We chose the possibly simplest parameterization of the solar differential rotation pattern of the form

$\Omega=\Omega_{\mathrm{eq}}\left(1-\alpha \cos ^{2} \psi\right)$.

$\Omega$ is the angular velocity at co-latitude $\psi, \Omega_{\mathrm{eq}}$ the equatorial angular velocity, and $\alpha$ a dimensionless parameter measuring the degree of differential rotation. $\alpha \approx 0.2$ for the Sun (see, e.g., Reiners \& Schmitt 2002). Knowing the stellar radius, the angular velocity can be transformed into a relation for the radial velocity as a function of position on the stellar disk. However, its complex functional dependence does not allow the expression of the rotation kernel by elementary functions analog to Eq. (5) (Huang 1961). We evaluated the kernels for individual $\mu$-rings numerically by discretizing the stellar disk employing polar coordinates. While seemingly straight-forward it proved difficult to obtain sufficient numerical accuracy, in particular at low radial velocities, and higher-than-expected resolution was necessary. We ensured numerically that the symmetry of the kernel functions with respect to the origin (i.e., zero radial velocity) was maintained so that no artificial line asymmetries were introduced in the resulting line profiles.

Figure 4 depicts an example comparing rotationally broadened profiles assuming rigid as well as differential rotation. We arbitrarily selected an Fe I line (at $6082 \AA$ ) of a 3D spectral synthesis calculation for the Sun, and solar-like rotational parameters, $V=1.8 \mathrm{~km} \mathrm{~s}^{-1}, \sin (i)=1.0$, and for the case of differential rotation $\alpha=0.2$. The plot shows that - as expected - our procedure of integrating over $\mu$-circles (here $N_{\mu}=4$ ) leaves no obvious imprint of spikes in the resulting profiles. Moreover, noticeable differences are present for fixed $V$ and $\sin (i)$. One might interpret the smaller degree of broadening in the differentially rotating case as simply a result of the the smaller disk-averaged

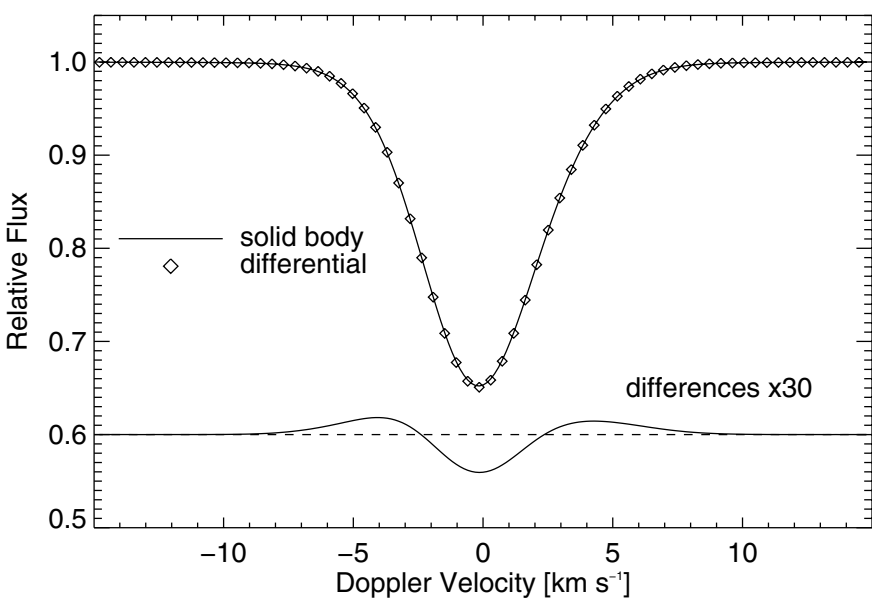

Fig. 4. Comparison of rotational broadening assuming a rigidly (solid line) and differentially (diamonds) rotating Sun. Thirty times enlarged differences of the resulting profiles (in the sense differential-rigid) are plotted in the lower part of the panel. For further explanations see text.

rotation rate for $\alpha>0$. However, from Eq. (6) we obtain for the root-mean-square radial velocity $v_{\mathrm{RMS}}$ due to the rotation over the stellar disk

$v_{\mathrm{RMS}}=\sqrt{\frac{2}{3}} V \sin (i) \sqrt{1-\alpha\left(\frac{3}{4} \cos (i)+\frac{1}{5} \alpha\right)}$

For our test case Eq. (7) gives a $0.4 \%$ smaller $v_{\text {RMS }}$ when $\alpha=0.2$ instead of zero. Changing the equatorial velocity $V$ by this amount assuming rigid rotation, leads to much smaller changes in the line profile than are visible in Fig. 4. However, for the particular Fe line it is possible to closely emulate its profile for differential rotation assuming rigid rotation where $V$ is $3 \%$ smaller than its nominal value.

\section{Conclusions}

The approach suggested in this paper provides an accurate treatment of rotational line broadening of a spherical star employing intensities at a modest number of limb-angles only. This is of practical importance in the context of 3D spectral synthesis calculations where angular resolution is computationally expensive. This also holds for differential rotation but the rotational kernel functions must be evaluated numerically. Our approach is not restricted to the $3 \mathrm{D}$ case but could be equally well applied in the standard 1D case. The issue is of course less pertinent in 1D since high angular resolution is affordable making the broadening method uncritical.

Acknowledgements. We thank Ansgar Reiners for helpful discussions about differential rotation. The work was funded by EU grant MEXT-CT-2004-014265 (CIFIST).

\section{References}

Caffau, E., Ludwig, H.-G., Steffen, M., et al. 2007, A\&A, in preparation Dravins, D., \& Nordlund, Å. 1990, A\&A, 228, 203

Gray, D. F. 1992, in The observation and analysis of stellar photospheres, 2nd ed. (Cambridge University Press)

Huang, S.-S. 1961, ApJ 133, 130

Reiners, A., \& Schmitt, J. H. M. M. 2002, A\&A, 384, 155 